\title{
An Interview with Professor Patrick DeLuca and Dr. Susan D'Souza on a Novel In Vitro Technique
}

\author{
Vivian Gray \\ Research Editor, Dissolution Technologies
}

\section{Introduction:}

merging special dosage forms and the development of

- in vitro release testing that accompanies these dosage

forms is of great interest to the dissolution analyst. An

AAPS Workshop co-sponsored with FDA titled Dissolution/In Vitro Release Testing and Specifications for Special Dosage Forms was held in September 2002 and included many presentations on new dosage forms. Dr. Patrick DeLuca's presentation was most interesting to me, as he described novel in vitro release testing techniques that appeared to be opening a new venue for testing methodology. Dr. DeLuca has published several articles that explore novel dosage forms, such as microspheres containing peptides in a controlled release matrix. In the following interview, Dr. Patrick DeLuca and Dr. Susan D'Souza, who worked extensively on this project, graciously answered my questions about novel dosage forms, in vitro release testing, and accelerated in vitro testing. The two apparatus discussed are shown in Figures 1 and 2 from the article"Methods to assess in vitro drug release from injectable polymeric particulate systems", Pharmaceutical Research, Vol. 23, No. 3. March 2006 (reprinted herein with permission from the Editor).

\section{$Q$ Inoted with interest in your article, "A novel in vitro technique for peptide-containing biodegrad- able microspheres" [1] the diagram of a dialysis setup (Figure 1). What led you to use this apparatus and what other apparatus had you used previously in studying these unique dosage forms?}

A We will be happy to discuss our work and publication. We selected the dialysis technique for two reasons. This allowed creating a sufficient sink while not agitating the microspheres. The latter was to simulate the in vivo conditions when localized in a depot site; that is, there is no agitation in the tissue. We designed the system and put together the apparatus. We have had ideas on improving it for commercialization but have never contacted anyone about that. Since the publication, we have made a few modifications. During/since the timeline of the interview, there have been studies to improve the dialysis apparatus. These studies have been published in AAPS PharmSciTech, Vol. 6 (2), 2005, article 42 as 'Development of a Dialysis In Vitro Release Method for Biodegradable Microspheres' (Figure 2) where a commercially available dialyzer was used. The main application of the dialyzer is purification of proteins, peptides, antibodies, et cetera, by using a membrane molecular weight cutoff (MWCO) such that small molecular weight contaminants such as buffer salts can easily pass through the membrane but the molecule of

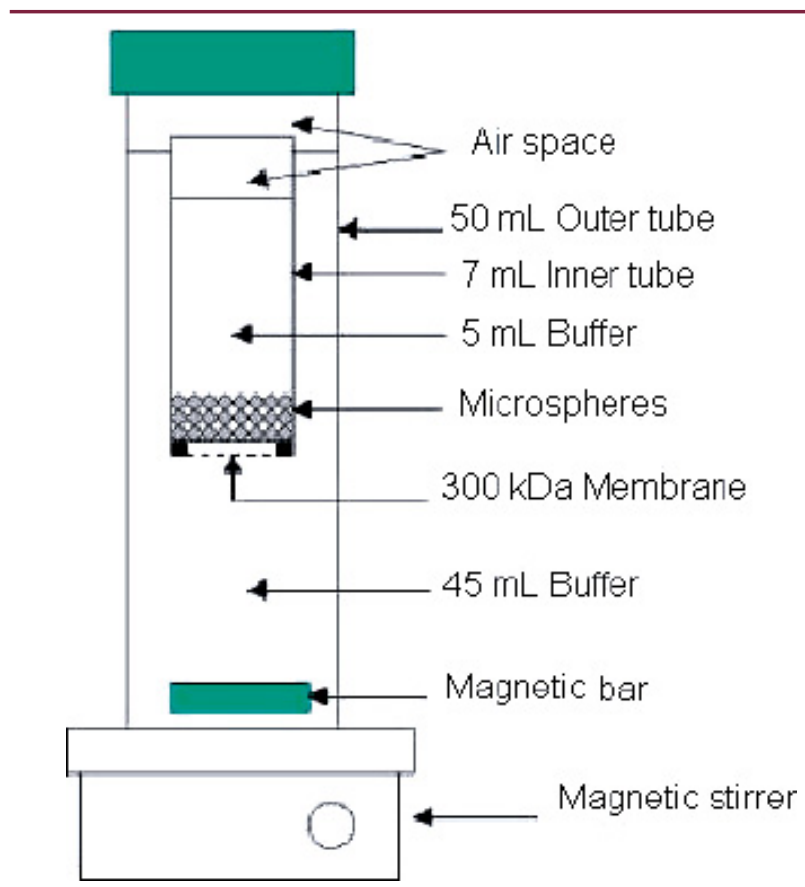

Figure 1: Dialysis method utilizing membrane at one end (from Kostanski et al [1])

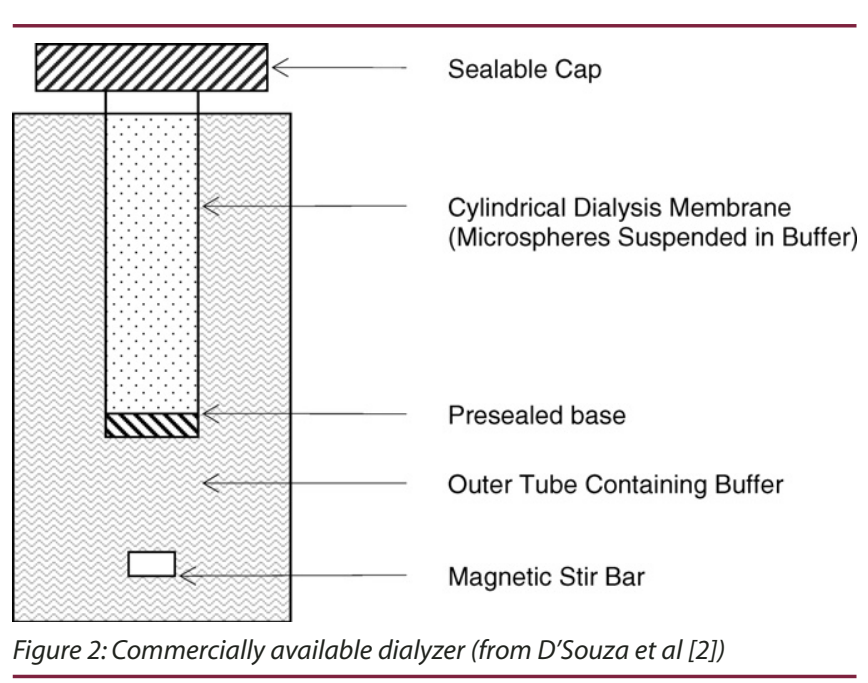

interest is retained. Reverse engineering the primary application resulted in passage of the drug molecule from the membrane into the outer media and allows for easy analysis.

Q I am sure our readers would like to have you describe why you chose this apparatus. What other apparatus did you try to work with before you chose 
this one, and what improvements do you anticipate in the future in the apparatus design?

A For parenteral dosage forms like microspheres, three methods have commonly been used to study release (review article published in Pharm. Res., Vol. 23 (3), 2006, 460-474). These include the 'sample and separate,"flowthrough cell,'and'dialysis' methods. Though all three methods may be used, the first two methods suffer from two major drawbacks:

1. Ease and/or mode of sampling ('sample and separate' method) along with rapid buffer replacement ('Flowthrough cell' method)

2. Set-up of apparatus ('Flow-through cell' method) With the dialysis method, sampling and buffer replacement is easy due to a physical separation of the dosage form from the outer media by a membrane. Also, using a commercially available apparatus resolves issues relating to set-up of the apparatus, such as user variability.

\section{$Q$ Could you describe the improvements of design from the one in the Figure 1?}

A Figure 2 shows the improved dialysis setup. The dialyzer is a commercially available product used to purify proteins and other molecules but has now been used to study drug release.

\section{$Q$ Why is the diffusion rate faster when not agitated? The microenvironment was more ideal without stirring - with agitation the diffusion or mixing was slower.}

A The dosage form (which is inside the dialyzer) is not subject to any agitation (similar to in vivo conditions as the dosage form is localized subcutaneously or intramuscularly). However, the outer media, which is continuously agitated, mimics blood flow. This ensures that sink conditions are maintained in vitro similar to in vivo conditions.

\section{$Q$ Can you comment on the quality and repro- ducibility of the dialysis membranes?}

A This is not an issue with the dialyzer as it is a commercial product, although for our use, we did check reproducibility and stability.

\section{$Q$ What are the controls from the manufacturer to assure batch-to-batch reproducibility? Are there quality tests?}

A The dialyzer is a commercial product, and the manufacturer has specifications for their intended purpose of the dialyzer, that is, protein separation. To ensure that this device could be used for our application, in vitro release testing, we performed various tests to assess stability and reproducibility of performance prior to use.

\section{$\mathrm{Q}$ How is the molecular weight cut-off of the membrane selected?}

A The MWCO has to be selected such that it allows for easy passage of the drug molecule to the outer sink.

$Q$ Could you comment on the aspects of accelerated conditions for dosage forms that have a long therapeutic affect?

A Using the dialyzer designed with a regenerated cellulose membrane, temperatures up to $60^{\circ} \mathrm{C}$ can be used for accelerated release studies (AAPS PharmSciTech, Vol. 6(4), 2005, article 70, selected for the 2005 AAPS Outstanding Graduate Student Research Award in Pharmaceutical Technologies). Also, the membrane can be washed and re-used, rendering it cost-effective.

\section{Q Could you describe the sampling steps?}

A Samples are withdrawn from the outer media. This eliminates the need for centrifugation, filtration, or destruction of microspheres, which are employed with the'sample and separate'method.

\section{Q Have you shown that there are no interferences from the media (placebo effect)?}

A Prior to starting an in vitro release study, it is important to know and understand media effects on release. Drug should be soluble and stable in the media and not interfere with subsequent analysis. In our studies, we did not find this to be the case.

\section{Q How reproducible and accurate are the results?}

A In our experience, results with the dialyzer have been reproducible (SD $= \pm 5 \%)$.

\section{$Q$ Is there a lag time?}

A With the previous apparatus (Figure 1), lag time was an issue due to the small surface area of the membrane used. However, with the dialyzer (Figure 2), this was significantly reduced (to 6 hours) due to the increased surface area. With any dialysis apparatus, lag time will be an issue and may be overcome by increasing the membrane surface area.

Q USP is now quite interested in performance tests (or in vitro release) for all products. What do you think of this activity and do you think this can be done? What about IVIVC testing? Is it a worthwhile pursuit for these special dosage forms? 
A Traditionally, in vitro tests have been used for quality control and IVIVC testing. However, with the advent of novel dosage forms, especially those that release the active over an extended period of time (weeks to months), in vitro release testing, especially under accelerated conditions, will aid the formulator by expediting product design and development while reducing costs associated with in vivo studies. It would be prudent to focus on designing an appropriate in vitro release test for the dosage form in question and glean as much information from the test as possible. However, the ultimate criterion for selection of an in vitro release method should rest on its ability to predict in vivo performance.
We at Dissolution Technologies appreciate the time Prof. DeLuca and Dr. D'Souza have spent giving this interview and know that our readers will find the information very interesting.

\section{References}

[1] J.W. Kostanski, P.P. DeLuca, A Novel In Vitro Release Technique for Peptide Containing Biodegradable Microspheres, AAPS PharmSciTech 2000, 1, article 4.

[2] S.S. D'Souza, P.P. DeLuca, Development of a dialysis in vitro release method for biodegradable microspheres, AAPS PharmSciTech 2005, 6, article 42. 